\title{
SITUACIÓN ACTUAL Y PERSPECTIVASDE LA GANADERÍA LECHERA EN LA CUENCA DE LIMA
}

\author{
M áximo Gamarra R. ${ }^{1}$
}

\section{Sector Lechero M undial}

El sector lechero, progresivamente, está integrando la economía gl obal; caracterizada por las exportaciones-importaciones privadas de productos, menos intervención de los gobiernos y las inversiones foráneas en la industria láctea.

Estecambiante ambientecrea amena zas y también oportunidades. Para mejor entendimiento del impacto de la liberalización global del comercio sobre la competitividad delalechería peruana en estos mercados, no podemosignorar las consecuencias del GATT y los Acuerdos dela Ronda del Uruguay que están cambiando toda la situación.

Producción Mundial

De acuerdo a la información de la FAO la población total de bovinos en el año 2000 fue de 1,350'130,000 y la producción de leche es de 484'895,261 TM (Anexo № 01). Losinventarios devacas lecheras delos principales países se muestran en el Cuadro 1.

Los principal es productores delecheson los países de la Comunidad Europea (CE), siguiéndoleen orden deimportancia Estados Unidos, India, Rusia, Brasil (enAméricadel Sur) y Nueva Zelanda en Oceanía.

El crecimiento mundial de 1999 sobre 1998 fue de 1\%. En el 2000, I la misma FAO publ ica una menor producción, sin comentar el cambio decifras. Los países que registran mayor crecimiento sonEE.UU., Brasil, Australia y Nueva Zelanda. Los países que decrecen son India y Pakistán.
La producción deEuropa (CE, 15 países) no cambia porque está regulada. En Oceanía, Nueva Zelanda y Australia producirán más leche por la sustitución de ganado ovino por bovino, debido a la baja rentabilidad por crisis del precio delanay por la facilidad decambiar porqueambas especies consumen el mismo forraje.

La producción en los países en desarrollo deAsiay América Latinacontinúacre ciendo. En la Comuni dad Andina de Naciones, Colombia y Ecuador han aumentado y son autosuficientes, y el Perú desde1992 está aumentando a un ritmo de $5 \%$.

\section{Consumo M undial}

El consumo mundial de lechey productos lácteos mantiene tendencia crecientelos últimos años. Deacuerdo a laFAO, el consumo por Regiones, el crecimiento del consumo de productos lácteos, en Amé rica Latina y Asia obedeceal rápido crecimiento de la población urbana con mejor capacidad de gasto. Los consumidores de todo el mundo tienen una amplia gama de productos lácteos a escoger.

El consumo por habitante varía según los tipos deeconomíay regiones del mundo. El consumo medio de países desarrollados bordea los 205 kg; en cambio, en los países en desarrollo, el consumo medio solo al canza 37 kg; en éstos países la demanda continúa aumentando, pero queda todavía una enorme brecha por Ilenar. El consumo medio en los países andi nos es de88 litros, mientras queel recomendado por la FAO asciende a $120 \mathrm{lt}$ por habitanteal año.

\section{${ }^{1}$ Fongal-Lima}


Cuadro 1. Población bovina productora de leche (en millones decabezas)

\begin{tabular}{|c|c|c|c|c|c|c|}
\hline & 1994 & 1995 & 1996 & 1997 & (p)1998 & Part\% 98 \\
\hline \multicolumn{7}{|l|}{$\begin{array}{l}\text { AMÉRICA DEL } \\
\text { NORTE }\end{array}$} \\
\hline Canadá & 1,224 & 1,224 & 1,237 & 1,253 & 1,242 & 1.0 \\
\hline México & 6,480 & 6,440 & 6,440 & 6,500 & 6,600 & 5.1 \\
\hline Estados Unidos & 9,500 & 9,458 & 9,361 & 9,258 & 9,190 & 7.1 \\
\hline Sub Total & 17,204 & 17,142 & 17,038 & 17,011 & 17,032 & 13.1 \\
\hline \multicolumn{7}{|l|}{ AMÉRICA DEL } \\
\hline Argentina & 2,300 & 2,350 & 2,300 & 2,400 & 2,500 & 1.9 \\
\hline Brasil & 17,500 & 17,500 & 17,100 & 17,067 & 16,900 & 13.0 \\
\hline Penú & 543 & 580 & 553 & 575 & 610 & 0.5 \\
\hline SubTotal 1/ & 21,813 & 21,860 & 21,181 & 21,367 & 21,405 & 16.5 \\
\hline \multicolumn{7}{|l|}{ EUROPA } \\
\hline Francia & 4,615 & 4,754 & 4,672 & 4,562 & 4,453 & 3.4 \\
\hline Alemania & 5,301 & 5,273 & 5,229 & 5,195 & 5,091 & 3.9 \\
\hline Italia & 2,287 & 2,167 & 2,100 & 2,100 & 2,100 & 1.6 \\
\hline Reino Unido & 2,318 & 2,268 & 2,130 & 2,090 & 2,060 & 1.6 \\
\hline Polonia & 3,866 & 3,715 & 3,442 & 3,550 & 3,496 & 2.7 \\
\hline Rusia & 19,800 & 18,400 & 17,450 & 15,900 & 14,500 & 11.2 \\
\hline Ucrania & 8,078 & 7,818 & 7,200 & 6,265 & 6,250 & 4.8 \\
\hline SubTotal 1/ & 56,924 & 54,904 & 52,560 & 49,845 & 48,033 & 37.0 \\
\hline \multicolumn{7}{|l|}{ ASIA } \\
\hline India & 31,500 & 33,000 & 33,500 & 34,500 & 35,000 & 26.9 \\
\hline China & 2,018 & 2,252 & 2,071 & 2,120 & 2,170 & 1.7 \\
\hline Japón & 1,052 & 1,034 & 1,035 & 1,032 & 1,022 & 0.8 \\
\hline Sub Total & 34,570 & 36,286 & 36,606 & 37,652 & 38,192 & 29.4 \\
\hline \multicolumn{7}{|l|}{ OCEANÍA } \\
\hline Australia & 1,762 & 1,786 & 1,822 & 1,977 & 2,002 & 1.5 \\
\hline Nueva Zelanda & 2,808 & 2,994 & 3,153 & 3,260 & 3,270 & 2.5 \\
\hline Sub Total & 4,570 & 4,780 & 4,975 & 5,237 & 5,272 & 4.1 \\
\hline TOTAL 1/ & 135,081 & 134,972 & 132,460 & 131,112 & 129,934 & 100.0 \\
\hline
\end{tabular}

1/ Se incluye en América del Sur: Chile y Venezuela; en Europa: Austria, Bélgica, Dinamarca, Finlandia, Grecia, Irlanda, Noruega, Portugal, España, Suiza, Suecia y Rumania. (p) - preliminar.

Fuente: Dairy World Market and Trade. 1988 
Cuadro 2. Producción mundial de lechefresca (en miles de TM)

\begin{tabular}{|c|c|c|c|c|c|c|c|c|}
\hline № & País & 1992 & 1993 & 1994 & 1995 & 1996 & 1997 & 2000 \\
\hline 1 & $\begin{array}{l}\text { EEUU } \\
\text { India }\end{array}$ & 3 & 6 & 1 & 0 & & & \\
\hline & Federación & 7015 & 46,2 & & & & & \\
\hline & Ale & & & & & & & 28,420 \\
\hline 4 & $\begin{array}{l}\text { Franc } \\
\text { Pakis }\end{array}$ & & & 2 & & & & $\begin{array}{r}24,890 \\
8,000\end{array}$ \\
\hline & Brasi & & & & & & & 22,134 \\
\hline & & & & & & & & \\
\hline 17 & Aus & & & & & & & 11,183 \\
\hline 18 & & & & & & & & \\
\hline 30 & Col & & & & & 5 , & 5,408 & 5,740 \\
\hline Te & & & & & & & & \\
\hline 47 & & & & & & & 1,929 & 1,995 \\
\hline 54 & & & & & 1,2 & & 1,348 & 1,421 \\
\hline 61 & Pen & 768 & 803 & 830 & 858 & 905 & 948 & 1,048 \\
\hline
\end{tabular}

Fuente: FAO

Cuadro 3. Consumo de leche fluida por regiones (en miles de TM)

\begin{tabular}{lrrrrrr}
\hline Región & 1995 & 1996 & 1997 & 1998 & $\begin{array}{r}1999 \\
\text { Estimado }\end{array}$ & $\begin{array}{r}2000 \\
\text { Pronóstico }\end{array}$ \\
\hline Amárica de Norte & 32,827 & 33,126 & 33,216 & 33,156 & 33,517 & 33,755 \\
Amárica de Sur & 13,940 & 14,618 & 15,513 & 16,374 & 16,619 & 16,930 \\
Unión Europea & 34,233 & 33,942 & 32,507 & 32,069 & 31,902 & 31,769 \\
Federación de Rusia & 18,125 & 17,500 & 17,315 & 17,615 & 17,600 & 17,635 \\
Asia & 36,233 & 38,527 & 38,292 & 41,177 & 40,753 & 41,343 \\
Oceanía & 2,319 & 2,398 & 2,034 & 2,320 & 2,330 & 2,340 \\
\hline Mundial & 148,166 & 150,660 & 151,281 & 151,501 & 152,581 & 153,512 \\
\hline
\end{tabular}

Cuadro 4. Consumo anual per cápita de lechefluida en al gunos países deAmérica Latina

\begin{tabular}{lc}
\hline País & Litros/Hab. \\
\hline Colombia & 114.0 \\
Venezuela & 95.2 \\
Ecuador & 110.9 \\
Bolivia & 32.8 \\
Chile & 125.9 \\
Perú & 53.5 \\
\hline Recomendado por la FAO & 120.0 \\
\hline
\end{tabular}

Fuente: FAO 


\section{M ercado M undial}

Durante los noventa, la evolución de la economíaen el mundo continuó condicionada por la política macroeconómica; lamayoría de países preocupados por mantener $\mathrm{d}$ equilibrio fiscal, bajar la inflación y promover $\mathrm{e}$ ahorro, contribuyendo a deprimir las tasas de crecimiento con el consecuente efecto sobre la di námica del mercado mundial para los diferentes productos lácteos.

El gobierno delos EE.UU encargó en 1998al Departamento deAgriculturay laUniversidad deWinsconsin-Madison elaborar un Análisis Económico de los Efectos de la Liberal ización del Comercio del Sector Leche ro Mundial. Este estudio fue presentado en Seattle(Washington), en Octubre18 de1999, desenvolviéndoselaliberalización del comercio mundial, especial menteel cumplimiento del GATT y Acuerdo de la Ronda del Uruguay, etc. cambiando la situación. El sector lechero progresi vamente está integrando la economía global lechera caracterizada por exportación-importación privada, menor intervención de los gobiernos y adicional inversión extranjera en la industria lechera.

El objetivo principal del estudio es evaluar los impactos dela liberalización, especial mente del GATT y Ronda del Uruguay sobre el mercado lechero mundial, luego del sector lechero de los EE.UU. Nosotros comentamos, especial mente, el impacto de los precios sobrel os precios de leche en puerta de establo.

El precio base de leche fresca en puerta de establo es el promedio de los países conformantes de cada bl oque, obtenidos con minuciosos cál cul os, sobreel cual secal cula los precios GATT 2000, los GATT 2005y el impacto “Libre Mercado" el año 2005.

Los países con precio superior al promedio bajarán al promedio, y los países con precio actual bajo subirán hastael promedio, como se nota en los bloques de Europa del Estey Ex-Soviéticos, Oceaníay Sudamérica.
Bajarán los países de Europa Occidental, Asia, Canadáy México. EE.UU. y otros países con precios cercanos al promedio actual se mantendrán con los mismos precios.

\section{El Mercado Peruano de Lácteos}

\section{Capacidad de Producción}

El inventario devacas en ordeño en el año 2000 es de 578,000 cabezas, equival ente al $12 \%$ del hato nacional. La mayor producción de leche fresca en el año 2000, se localiza en las cuencas lecheras de Arequipa, Cajamarca y Lima.

Las diferencias enla producción delas distintas cuencas obedecen a los diferentes sistemas de producción y desigual dades tecnológicas. Las zonas cercanas a Lima, Arequipa y Cajamarca presentan un nivel tecnológico superior con relaciónal resto del país.

Producción de Lima

El inventario devaca en producción al año 2000 se estima en 50,000. Los índices de producción corresponden a los sistemas de producción claramente definidos. Losíndices de producción del sistema estabulado, según el control mensual de producción del Servicio Oficial deProductividad Lechera se indican en los cuadros 8 y 9 . Esta población devacas con registros de producción es partede una mayor canti dad sin registro, aproximadamente con los mismos indices de producción.

La mayor población se cría bajo el sistema de los pequeños productores, al pastoreo en la si erra de Lima y mixtos pastoreo-concentrados en los valles de la costa. Losíndices productivos son los del promedio nacional.

M ercado

Limaes el mercado principal del Perú; el producto más consumido es la leche eva 
Cuadro 5. El impacto de los precios de la lecheen puerta de establo

\begin{tabular}{lccccc}
\hline & \multicolumn{2}{c}{$\begin{array}{c}\text { Precio base dela } \\
\text { leche }\end{array}$} & $\begin{array}{c}\text { GATT } \\
\text { 2000 }\end{array}$ & $\begin{array}{c}\text { GATT } \\
\text { 2005/Todo }\end{array}$ & "FreeTrade" \\
& \$US/MT & \$US/cwt & \multicolumn{3}{c}{$\begin{array}{c}\text { \% Cambio } \\
\text { dela base }\end{array}$} \\
\hline Europa Occidental & 412 & 18.70 & -5.5 & -13.5 & -25.8 \\
Europa del Este & 191 & 8.66 & 1.6 & 6.9 & 25.9 \\
Asia Oriental & 455 & 20.66 & -0.7 & -0.3 & -26.4 \\
Japón & 709 & 32.18 & -1.5 & -1.6 & -36.4 \\
SudesteAsiático & 219 & 9.95 & 0.0 & -0.3 & 0.2 \\
OrienteMedio/África del Norte & 381 & 17.30 & 0.1 & 1.2 & 0.5 \\
Oceanía & 185 & 8.40 & 2.1 & 8.5 & 35.5 \\
Australia & 213 & 9.67 & -0.8 & 2.7 & 22.5 \\
Nueva Zelanda & 161 & 7.31 & 5.8 & 15.8 & 50.8 \\
America del Norte & 302 & 13.70 & -0.7 & -1.0 & -4.9 \\
Canadá & 414 & 18.79 & -2.0 & -3.5 & -32.3 \\
USA & 289 & 13.12 & -0.5 & -0.6 & -0.4 \\
Centro y Sud América & 287 & 13.04 & 1.8 & 0.9 & -6.1 \\
México & 342 & 15.52 & -0.3 & -4.08 & -17.3 \\
SAM, N & 300 & 13.62 & 0.8 & -0.5 & -7.8 \\
SAM, S & 200 & 9.08 & 9.6 & 15.1 & 17.2 \\
Resto del Mundo & 376 & 17.05 & -0.1 & 1.5 & 0.4 \\
\hline Promedio Mundial & 297 & 13.49 & -1.6 & -3.5 & -7.1 \\
\hline
\end{tabular}

Cuadro 6. Perú: vacas en ordeño, producción de lechey rendimiento (1990-2000)

\begin{tabular}{lrrrrrrr}
\hline & 1990 & 1993 & 1994 & 1995 & 1996 & 1997 & 2000 \\
\hline $\begin{array}{l}\text { Vacas en ordeño (Miles de } \\
\text { unidades) }\end{array}$ & 592 & 553 & 563 & 564 & 553 & 526 & 578 \\
$\begin{array}{l}\text { Producción (Miles deTM) } \\
\text { Rendimiento (Kg/vaca/año) }\end{array}$ & 777 & 803 & 830 & 858 & 905 & 948 & 1066 \\
& 1313 & 1452 & 1474 & 1521 & 1637 & 1802 & 1844 \\
\hline
\end{tabular}

Desde 1996 se considera Cajamarca como la suma de las subregiones agrarias Chota, J aén y Cajamarca.

Fuente: Ministerio de Agricultura

Cuadro 7. Producción de leche por principales departamentos (en miles de TM)

\begin{tabular}{lcccccccc}
\hline & 1990 & 1993 & 1994 & 1995 & 1996 & 1997 & 1998 & 2000 \\
\hline Arequipa & 167 & 178 & 175 & 195 & 202 & 207 & 214 & 245,264 \\
Cajamarca & 90 & 88 & 113 & 115 & 113 & 158 & 169 & 153,603 \\
Lima & 100 & 83 & 91 & 104 & 119 & 117 & 129 & 153,780 \\
\hline Perú & 777 & 803 & 830 & 858 & 905 & 948 & 998 & 1066,955 \\
\hline
\end{tabular}

Fuente: Ministerio de Agricultura

Elaboración: Base de Datos Fundación Perú 
Cuadro 8. Rendimiento en los principales departamentos productores de leche (vacas en producción 1997-2000)

\begin{tabular}{lrr}
\hline & \multicolumn{2}{c}{$\mathrm{Kg} /$ vaca/año } \\
\cline { 2 - 3 } & 1997 & 2000 \\
\hline Arequipa & 3,090 & \\
Cajamarca & 1,816 & 3,075 \\
Lima & 2,854 & 50,000 \\
\hline Total Nacional & 1,802 & \\
\hline
\end{tabular}

Cuadro 9. Servicio oficial de productividad lechera: Lima

\begin{tabular}{ccccc}
\hline Año & $\begin{array}{c}\text { № } \\
\text { Establos }\end{array}$ & $\begin{array}{c}\text { № } \\
\text { Vacas }\end{array}$ & $\begin{array}{c}\text { Promedio diario general } \\
\text { deleche }(\mathrm{kg})\end{array}$ & $\begin{array}{c}\text { Promedio vacas en ordeño } \\
\text { leche }(\mathrm{kg})\end{array}$ \\
\hline 1999 & 41 & 6,734 & 20.2 & 23.4 \\
\hline
\end{tabular}

Cuadro 10. Perú: importación de lecheen 1998

\begin{tabular}{lrrr}
\hline & & \multicolumn{2}{c}{ Factor de equivalencia } \\
\cline { 3 - 4 } \multicolumn{1}{c}{ Producto } & TM & $\begin{array}{c}\text { Conversión a } \\
\text { lechefluida }\end{array}$ & $\begin{array}{c}\text { En leche } \\
\text { fluida (TM) }\end{array}$ \\
\hline Lecheen Polvo LEP & 26,718 & 10 & 267,180 \\
Lecheen Polvo LPD & 15,325 & 8.45 & 129,498 \\
LecheEvaporada y otros & 6,720 & 2 & 13,440 \\
& 5,402 & 8.79 & 47,483 \\
& 5,660 & 10 & 56,600 \\
\hline Total importación delechefluida & 61,003 & & 516,026 \\
\hline
\end{tabular}

Los datos de factor de conversión son de Fundación Perú.

Fuente: Ministerio de Agricultura

poraday lasiguen en importancia, lechepasteurizada, condensada, polvoy maternizada. De los derivados lácteos y en orden de importanciasonel yogurt, queso, cremay mantequilla.

Paracubrir sus requerimientosel Perú importa; en 1998importó 516,026 TM enequival ente delechefluida. Nueva Zelanda elrlanda fueron los países de procedencia más importantes.
Precios

Los precios pagados al productor por lechefresca enfriada puesto en planta industrial, convertidos en US dólar ha decrecido losúltimos 4 años.

La mediana y pequeña industria, los programas social es pagados con dinero del tesoro públi ico han incrementado los precios cuando la ol igopólica gran industriaen soles 
Cuadro 11. Precio promedio anual pagado al productor (en planta)

\begin{tabular}{lccc}
\hline Año & $\begin{array}{c}\text { Nacional Minag } \\
\text { US } \$ \mathrm{~kg}\end{array}$ & $\begin{array}{c}\text { Lima } \\
\text { US\$ kg }\end{array}$ & Incremento Lima \% \\
\hline 1994 & 0.250 & 0.348 & \\
1995 & 0.268 & 0.329 & -5.50 \\
1996 & 0.268 & 0.339 & 3.20 \\
1997 & 0.252 & 0.328 & -3.29 \\
1998 & 0.250 & 0.325 & -0.78 \\
1999 & 0.234 & 0.292 & -10.14 \\
2000 & 0.234 & 0.286 & -2.20 \\
\hline
\end{tabular}

Cuadro 12. Precio al consumidor: Lima

\begin{tabular}{lll}
\hline & \multicolumn{2}{c}{ Kilo } \\
\hline Lecheevaporada & S/ 2.41 & US $\$ 0.69$ \\
Lechefresca pasteurizada & S/.2.37 & US $\$ 0.68$ \\
\hline
\end{tabular}

Fuente: MINAG

corrientes no ha incrementado los úl timos 40 meses, lo quesignifica queen sol constante o su equival ente, ha di smi nuido.

Por otro lado el precio al consumidor final es de S/. 2.41 por leche evaporada y S/. 2.37 paralechefresca pasteurizada o sea US\$ 0.69 y US\$ 0.68 .

Según la SIAG del MINAG, referidos al estudio de Wisconsin-Madison, Ios precios al productor deUS $\$ 0.286$ estámuy cercano al promedio mundial de US\$ 0.297 .

\section{Perspectivas de la Lechería en Lima}

Un análisis de Debilidades, Fortalezas, Amenazas y Oportunidades nos puede ayudar para predecir un escenario al año 2006. Interpretar el estudio de Winsconsin- Madison anivel mundial nos relacionará con el mercado mundial, sobre todo en un escenario de mercado libre (Free Trade) en el mismo año 2006.
- Debilidades

- Deficienciasentecnología nutrición, calidad de leche, acopio, transporte (carreteras) y manejo demateria prima.

- Estructuraproductiva, pequeñapropiedad de tierras y ganado.

- El nivel deingreso delos consumidores.

- El 75\% de la producción nacional se consume como leche fresca.

- Lapresenciaoligopólicadelagranindustria queconcentra en una sola empresa más del $70 \%$ del negocio.

- El veto sanitario por no ser pás libre de enfermedades como la fiebre aftosa.

- Fortalezas

- La experiencia para manejar distintos sistemas de producción en costa, sierra y selva.

- La experiencia de sobrevivir a las más adversas condiciones decontrol deprecios, terrorismo, enfermedades, importaciones doblementesubsidiadas. 
- Desarrollo de ganadería en condiciones ambientales muy variadas y al ejadas delos puestos y centros de producción.

- Amenazas

- Inestabilidad dela macroeconomía

- Deterioro de la demanda por falta de capacidad adquisitiva.

- La debilidad del sector para negociar aranceles, tarifas con otros sectores de la economía.

- Desorganización de los productores. Facilidad paranacer y desaparecer gremios einstituciones.

- Debilidad del sector y los productos para negociar ene CAN, MERCOSUR, ALCA y otros como los bilaterales de país a país.

- Ingreso a los países del CAN deproductos a precios dereal ización y su triangul ación subsiguiente.

- Oportunidades

- Capacidad de producción y autoabastecimiento.

- El mercado de los programas sociales que puedepermitir el desarrollo del sector lechero hasta niveles de abastecimiento.

- La OMC tiene como objetivo eliminar gradualmente las barreras. El 95\% de la leche tiene comercio intrazonal o de autoabastecimiento. El 5\% seexporta-importay llegaránal promediomundia comosehavisto enel respectivo cuadro. Subirán sus precios hasta el promedio los páses como Nueva Zelanda, Australia, Europa del Este, Rusia, Unuguay y Argentina, y bajaránal promedio Unión Europea, Japón, Canadá y México. Los otros páses están cerca al promedio.

- El MERCOSUR para el Perú trae más riesgos que oportunidades, aunque Brasil es deficitario, es natural que Uruguay y Argentina deseen complementar sus mercados con Perú y Venezuela que son tambiénimportadores.

\section{Conclusiones}

- La lechería en Lima tiene 2 niveles diferenciados. Requiere que el sector de al ta producción transmitasu tecnol ogíaal otro sector.

- Los productores deben tomar conciencia del nuevo entorno. Agruparse, particularmente los pequeños, es la mejor manera de hacer economías de escala.

- Todos los productores deben dar total respal do a sus instituciones paraal canzar una gran representación ante el Estado y la granindustria.

- La pequeña y mediana industria deben complementar e impulsar la cadena productiva láctea, con marca propia.

- El Perú, especialmenteLima, seesperaque aumentesu consumo dederivados lácteos como quesos, yogurt, helados y otros con menor contenido de grasas.

- Es necesario al canzar la categońa de país libre de aftosa

- Para alcanzar la condición de país autosuficiente es necesario importar va cas para la costa y mejorar agresivamentelas condiciones de la sierra y selva.

- Tan importante como la productividad es la calidad de la leche.

- Esimpostergablela capacitación detodos los ganaderos; una forma será la formación de Grupos de auto-desarrollo. Para esto será necesario enviar a los jóvenes a capacitarse en los sistemas de producción más parecidos a Lima.

- El objetivo principal es lograr quelos productores observen quesu crecimiento está en lograr autoabastecimiento vía la mejora de productividad lechera de los hatos, aprovechar las economía de escal a agnt pándose convenientemente, para integrar cadenas con la industria en igualdad de condiciones porquelainversiónen el sector es aproximadamentede 2/3 los productores de leche y 1/3 la maquila. 
Anexo 1. Población bovina y producción de leche (kg) por país en el año 2000

\begin{tabular}{|c|c|c|}
\hline Pais & Produccion de leche fresca & Poblacion total de bovinos \\
\hline China & $7,873,750$ & $104,582,029$ \\
\hline Afganistán & $2,100,000$ & $3,477,550$ \\
\hline Albania & 815,000 & 720,000 \\
\hline Algeria & $1,000,000$ & $1,650,000$ \\
\hline American Samoa & 16 & 103 \\
\hline Angola & 191,000 & $4,042,000$ \\
\hline Antigua y Barbuda & 6,000 & 15,700 \\
\hline Argentina & $9,800,000$ & $55,000,000$ \\
\hline Armenia & 470,000 & 478,730 \\
\hline Australia & $11,183,000$ & $26,716,000$ \\
\hline Austria & $3,349,910$ & $2,150,000$ \\
\hline República Azerbayar & $1,010,810$ & $1,945,200$ \\
\hline Bahamas & 550 & 700 \\
\hline Bahrain & 14,001 & 11,000 \\
\hline Bangladesh & 755,000 & $23,652,000$ \\
\hline Barbados & 8,100 & 23,000 \\
\hline Belarus & $4,320,000$ & $4,325,700$ \\
\hline Belgium-Luxemburgo & $3,600,000$ & $3,085,000$ \\
\hline Belice & 7,200 & 59,000 \\
\hline Benin & 20,800 & $14,381,000$ \\
\hline Bermuda & 1,350 & 600 \\
\hline Bhutan & 28,990 & 435,000 \\
\hline Bolivia & 231,454 & $6,724,590$ \\
\hline Bosnia Herzegovina & 270,000 & 461,928 \\
\hline Botswana & 101,500 & $2,350,000$ \\
\hline Brasil & $22,134,000$ & $167,471,008$ \\
\hline Bulgaria & $1,200,000$ & 682,000 \\
\hline Burkina Faso & 162,998 & $4,704,138$ \\
\hline Burundi & 18,550 & 320,000 \\
\hline Camboya & 20,400 & $3,000,000$ \\
\hline Camerún & 125,000 & $5,900,000$ \\
\hline Canadá & $8,090,000$ & $12,786,000$ \\
\hline Cabo Verde & 5,100 & 21,500 \\
\hline Africa Central & 62,000 & $2,950,000$ \\
\hline Chad & 151,200 & $5,595,000$ \\
\hline Chile & $2,160,000$ & $4,068,000$ \\
\hline Colombia & $5,740,000$ & $26,000,000$ \\
\hline Comoro & 4,450 & 52,000 \\
\hline Combo den Republic of. & 5,200 & 822,355 \\
\hline Combo Republic of. & 1,000 & 77,000 \\
\hline Costa Rica & 707,084 & $1,715,386$ \\
\hline Cracia & 641,300 & 427,000 \\
\hline Cuba & 617,800 & $4,700,000$ \\
\hline Cyprus & 133,000 & 55,841 \\
\hline
\end{tabular}


Viene..

\begin{tabular}{|c|c|c|}
\hline País & Producción de leche fresca & Población total de bovinos \\
\hline Czech Republic & $2,708,120$ & $1,573,530$ \\
\hline Costa de Marfil & 24,000 & $1,350,000$ \\
\hline Denmark & $4,464,500$ & $1,850,000$ \\
\hline Guinea Ecuatorial & & 4,800 \\
\hline Faeroe Ecuatorial & & 2,000 \\
\hline Suam & & 100 \\
\hline Djibuti & 8,050 & 269,000 \\
\hline Dominica & 6,100 & 13,400 \\
\hline República Dominicana & 397,750 & $1,904,400$ \\
\hline Ecuador & $1,995,599$ & $5,109,800$ \\
\hline Egipto & $1,644,790$ & $3,180,000$ \\
\hline El Salvador & 400,814 & $1,212,200$ \\
\hline Eritrea & 41,000 & $1,800,000$ \\
\hline Estonia & 600,000 & 285,600 \\
\hline Etiopía & 930,000 & $35,000,000$ \\
\hline Islas Malvinas & 1,660 & 4,439 \\
\hline Isla Fiji & 57,600 & 350,000 \\
\hline Finlandia & $2,500,000$ & $1,086,800$ \\
\hline Francia & $24,890,000$ & $20,527,100$ \\
\hline French Guyane & 250 & 9,000 \\
\hline French Polinesia & 1,000 & 10,000 \\
\hline Gabón & 1,575 & 36,000 \\
\hline Gambia & 7,175 & 370,000 \\
\hline Gaza Strip Palestine & 8,000 & 3,200 \\
\hline Georgia & 721,000 & $1,122,100$ \\
\hline Germany & $28,420,000$ & $14,657,901$ \\
\hline Ghana & 33,410 & $1,285,000$ \\
\hline Grecia & 770,000 & 590,000 \\
\hline Granada & 520,000 & 4,380 \\
\hline Guadaloupe & 113 & 80,410 \\
\hline Guatemala & 320,000 & $2,300,000$ \\
\hline Guinea & 72,000 & $2,368,000$ \\
\hline Guinea-Bissau & 13,260 & 530,000 \\
\hline Guyana & 13,000 & 220,000 \\
\hline Haití & 41,250 & $1,430,000$ \\
\hline Honduras & 729,000 & $1,950,000$ \\
\hline Hungary & $2,091,000$ & 857,000 \\
\hline Island & 107,353 & 72,100 \\
\hline India & $30,900,000$ & $218,800,000$ \\
\hline Indonesia & 384,300 & $17,102,000$ \\
\hline Irán & $4,403,000$ & $8.100,000$ \\
\hline Irak & 318,750 & $1,350,000$ \\
\hline Ireland & $5,448,000$ & $6,707,500$ \\
\hline Israd & $1,194,000$ & 388,000 \\
\hline Italy & $11,741,000$ & $7,184,000$ \\
\hline
\end{tabular}


Viene.

\begin{tabular}{|c|c|c|}
\hline País & Producción de leche fresca & Población total de bovinos \\
\hline Jamaica & 53,000 & 400,000 \\
\hline J apón & $8,500,391$ & $4,588,000$ \\
\hline J ordan & 145,000 & 57,000 \\
\hline Kazakhstan & $3,600,000$ & $3,998,000$ \\
\hline Kenya & $2,250,000$ & $13,794,000$ \\
\hline Korea Republic People & 90,000 & 600,000 \\
\hline Korea Republic & $2,243,941$ & $2,486,495$ \\
\hline Kuwait & 41,409 & 20,400 \\
\hline Kgrgystan & $1,090,472$ & 932,273 \\
\hline Laos & 6,000 & 986,600 \\
\hline Micronesia & & 13,900 \\
\hline Latuia & 822,983 & 378,400 \\
\hline Lebanon & 205,000 & 77,000 \\
\hline Lezatho & 23,750 & 520,000 \\
\hline Liberia & 715 & 36,000 \\
\hline Libian Arab. & 135,000 & 143,000 \\
\hline Lechstentain & 12,000 & 6,000 \\
\hline Lituania & $1,560,000$ & 897,800 \\
\hline Macedonia & 180,000 & 290,000 \\
\hline Madagascar & 535,000 & $10,364,000$ \\
\hline Malawi & 35,000 & 770,000 \\
\hline Malaysia & 42,000 & 723,346 \\
\hline Mali & 151,900 & $6,200,000$ \\
\hline Malta & 47,969 & 19,200 \\
\hline Martinique & 2,060 & 30,000 \\
\hline Mauritania & 101,500 & $1,435,000$ \\
\hline Mauritius & 4,700 & 29,400 \\
\hline México & $9,474,480$ & $30,293,100$ \\
\hline Moldova Rep. & 550,000 & 416,000 \\
\hline Mongolia & 285,000 & $3,500,000$ \\
\hline Monserrat & 2,250 & 9,700 \\
\hline Morocco & $1,150,000$ & $2,664,600$ \\
\hline Mozambique & 60,180 & $1,320,000$ \\
\hline Myanmar & 611,908 & $10,964,043$ \\
\hline Namibia & 75,000 & $2,063,000$ \\
\hline Nepal & 337,455 & $7,030,698$ \\
\hline Netherland & $10,800,000$ & $4,200,000$ \\
\hline Netherland Antilla & 380 & 600 \\
\hline New Caledonia & 3,690 & 123,000 \\
\hline New Zealand & $12,014,000$ & $9,457,000$ \\
\hline Nicaragua & 230,636 & $1,660,000$ \\
\hline Niger & 168,000 & $2,217,000$ \\
\hline Nigeria & 385,875 & $19,830,000$ \\
\hline Niue & 50 & 112 \\
\hline Norway & $1,759,000$ & $1,041,600$ \\
\hline Oman & 19,110 & 149,000 \\
\hline Pakistán & $8,039,000$ & $22,000,000$ \\
\hline Panamá & 174,240 & $1,359,800$ \\
\hline
\end{tabular}


Viene..

\begin{tabular}{|c|c|c|}
\hline País & Producción deleche fresca & Población total de bovinos \\
\hline Papua-New Guinea & 130 & 87,000 \\
\hline Paraguay & 329,800 & $9,910,000$ \\
\hline Perú & $1,048,100$ & $4,903,000$ \\
\hline Philipines & 9,850 & $2,552,910$ \\
\hline Poland & $11,730,979$ & $6,082,549$ \\
\hline Portugal & $1,850,000$ & $1,245,000$ \\
\hline Puerto Rico & 357,115 & 388,307 \\
\hline Qatar & 11,200 & 14,200 \\
\hline Rumania & $4,500,000$ & $3,154,900$ \\
\hline Russian Federation & $31,560,000$ & $27,500,000$ \\
\hline Rioanda & 85,000 & 725,000 \\
\hline Reunion & 13,500 & 27,000 \\
\hline Singapore & & 200 \\
\hline Saint Kits and Nevis & & 3600 \\
\hline Saint Elena & & 6920 \\
\hline Saint Lucía & 1,100 & 12,400 \\
\hline Saint Vicent/Brenadine & 1,370 & 6,200 \\
\hline Samoa & 1,150 & 26,000 \\
\hline Sao Tome and Principe & 138 & 4,050 \\
\hline Saudi Arabia & 601,000 & 297,000 \\
\hline Senegal & 105,012 & $2,960,000$ \\
\hline Sychelles & 310 & 1,400 \\
\hline Sierra Leone & 21,250 & 420,000 \\
\hline Slovaquia & $1,099,399$ & 665,055 \\
\hline Slovenia & 634,448 & 461,425 \\
\hline Solomon 15 & 1,300 & 12,000 \\
\hline Somalia & 530,000 & $5,100,000$ \\
\hline Sudafrica & $2,667,000$ & $13,700,000$ \\
\hline Spain & $5,900,000$ & $6,203,000$ \\
\hline Sri Lanka & 220,000 & $1,616,700$ \\
\hline Sudán & $3,072,000$ & $37,093,000$ \\
\hline Suriname & 13,000 & 106,000 \\
\hline Swaziland & 35,000 & 610,000 \\
\hline Sweden & $3,300,000$ & $1,713,000$ \\
\hline Switziland & $3,910,000$ & $1,600,000$ \\
\hline Syrian Arab Rep. & $1,150,000$ & 920,000 \\
\hline Tajikistán & 305,500 & $1,042,000$ \\
\hline Tanzania & 685,000 & $14,380,000$ \\
\hline Tailandia & 468,543 & $6,100,000$ \\
\hline Togo & 6,975 & 215,000 \\
\hline Tonga & 225 & 9,318 \\
\hline Trinidad Tobago & 10,241 & 35,000 \\
\hline Tunisia & 890,000 & 790,000 \\
\hline Turkey & $8,800,000$ & $11,031,000$ \\
\hline Turkmenistán & 900,000 & 850,000 \\
\hline
\end{tabular}


Viene..

\begin{tabular}{lrr}
\hline País & Producción delechefresca & Población total de bovinos \\
\hline US Virgia Is. & 1,960 & 8,000 \\
Uganda & 511,000 & $5,965,500$ \\
Ucrania & $12,400,000$ & $10,626,500$ \\
Unit Arab Emirate & 8,700 & 110,000 \\
United Kingdom & $14,461,000$ & $11,133,000$ \\
USA & $76,294,000$ & $98,048,000$ \\
Uruguay & $1,421,749$ & $10,800,000$ \\
Uzbekistán & $3,692,000$ & $5,268,300$ \\
Vanuatu & 3,000 & 152,000 \\
Venezuela & $1,311,204$ & $15,800,000$ \\
Vietnam & 41,738 & $4,137,000$ \\
Wallis and Futuna Is. & 30 & 60 \\
West Bank & 26,800 & 11,658 \\
Yemen & 176,850 & $1,282,975$ \\
Yugoslavia & $1,825,000$ & $1,452,000$ \\
Zambia & 64,200 & $2,373,000$ \\
Zimbawe & 310,000 & $5,550,000$ \\
British Virgia Is. & & 2,400 \\
Brunei & & 2,200 \\
Cayman Is. & & 1,300 \\
Cook Is & & 120 \\
\hline TOTAL & & $1,350,130,000$ \\
& & \\
\hline
\end{tabular}

